\title{
Combination Therapy in Pulmonary Arterial Hypertension: Gleaning a Practical Approach from the Randomized Trials
}

\author{
David C. Booth, MD ${ }^{1}$ \\ ${ }^{1}$ Section of Cardiology, University of Kentucky Medical Center, \\ Lexington VA Medical Center, Lexington, Kentucky \\ Int J Angiol 2019;28:93-99.
}

\begin{abstract}
Address for correspondence David C. Booth, MD, Section of Cardiology, University of Kentucky Medical Center, Lexington VA Medical Center, 326 Wethington Building, 900 S. Limestone St, Lexington, KY 40536-0200 (e-mail: dcbooth@uky.edu).
\end{abstract}

\author{
Abstract \\ Keywords \\ - pulmonary arterial \\ hypertension \\ - combination PAH \\ drug therapy \\ - WHO Group 1 PAH \\ - macitentan \\ - ambrisentan \\ - tadalafil \\ - selexipag
}

In the past decade, combination therapy in pulmonary arterial hypertension (PAH) has evolved from something PAH practitioners felt almost compelled to do, notwithstanding the absence of data, to a strategy proven by well-conducted randomized clinical trials. Whereas in the past, PAH treatment was limited to parenteral epoprostenol; today multiple drugs administrable either parenterally, inhaled, or orally have expanded the options for treating PAH patients. The SERIPHIN, AMBITION, and GRIPHON trials and emerging findings in FREEDOM-EV confirm the validity of a combined-therapy approach. Data from these trials in which either combined therapy was planned or an agent was added to background therapy have demonstrated significant reduction in the progression of disease and are on the cusp of demonstrating survival benefit. Combination therapy may be started simultaneously in some cases, but in many cases a stepped approach to initiating a second, or third, agent is better tolerated. Trials of all the specific combinations of drugs may not be possible, but a continuing trend toward treating PAH with multiple agents is likely. Currently, Food and Drug Administration-approved agents are predominantly pulmonary vasodilators acting through different pathways, with minimal impact on progression of the proliferative pulmonary arteriopathy that is the key pathologic finding in PAH. It is to be hoped that treatment strategies that result in halting progression and substantial reversal of pulmonary arteriolar obstruction will soon be discovered and available.
World Health Organization (WHO) Group 1 pulmonary arterial hypertension (PAH) patients in whom pulmonary vasodilator testing reveals a striking vasodilator response, and therefore can be treated with high-dose generic calcium channel blocking agents, constitute less than $10 \%$ of $\mathrm{PAH}$ patients ${ }^{1}$ and are not the subject of this review. At opposite end of the spectrum of disease severity, with the advent of newer oral therapies, initial parenteral treatment of PAH has come to be reserved predominantly for functional Class IV patients. With the earliest reports suggesting possible benefit, ${ }^{2}$ combination therapy was being employed in PAH for more than a decade before prospective randomized controlled trials demonstrated efficacy. ${ }^{3-5}$ In such an implacable disease, and still lacking an effective antiproliferative remedy, ${ }^{1}$ the clinician felt impelled to do everything that was reasonable for the patient, perhaps especially in young persons. Among other data, an open-label trial of ambrisentan $5 \mathrm{mg}$ daily for 24 weeks added to background therapy demonstrated a significant increase in 6-minute walk distance (6MWD) of $21 \mathrm{~m}$ and a decrease in natriuretic peptide type B. ${ }^{6}$ Meanwhile, important voices in PAH were highlighting the need for end point-driven studies. ${ }^{7}$ Confounders to combination therapy in PAH include that not all randomized trials of combination therapy have been positive, ${ }^{8}$ and at times in the United States, choice of therapy can be governed as much by the payer as the clinician. The ethical 
impasse in PAH to a placebo control group and sample size requirements are among impediments to conclusively demonstrating survival benefit, leaving the epoprostenol trial of Barst et $\mathrm{al}^{9}$ as the only published randomized trial in PAH to conclusively demonstrate survival benefit thus far. Approved inhaled and oral therapies targeting not only the IP receptor but also drugs targeting the endothelin and the nitric oxide pathways have resulted in a paradigm shift in PAH management for all but functional Class IV patients, and a not-insignificant number of the latter, once stabilized seem capable of being weaned off parenteral therapy once oral and/or inhaled therapies are initiated. A critical review of randomized trial findings can facilitate an understanding of when and how to initiate combination therapy in PAH at a practical level. However, development of more precise definitions of PAH and stages of disease ${ }^{10}$ may be necessary for effective application of therapy.

\section{Randomized Trials}

\section{SERAPHIN}

The SERAPHIN trial, ${ }^{3}$ a multicenter, double-blind trial assessing the impact of the nonselective oral endothelin receptor antagonist macitentan on morbidity and mortality, was the first randomized trial of sufficient sample size to signal that combination therapy could result in a decrease in progression of disease. Patients in functional Class II, III, or IV who had idiopathic or heritable $\mathrm{PAH}$ or $\mathrm{PAH}$ associated with connective tissue disease, repaired congenital systemic-topulmonary shunts, human immunodeficiency virus infection, or drug use or toxin exposure were eligible for the trial. The trial was described as placebo controlled, but concomitant treatment with oral phosphodiesterase type 5 (PDE-5) inhibitors, oral or inhaled prostanoids, calcium-channel blockers, or L-arginine was permitted if the patient had been receiving a stable dose for at least 3 months before randomization. Patients receiving intravenous or subcutaneous prostanoids were excluded. Patients were enrolled at 151 centers in 39 countries between May 2008 and December 2009. A total of 742 patients were randomly assigned to placebo (250 patients), macitentan $3 \mathrm{mg}$ (250), or maciten$\tan 10 \mathrm{mg}$ (242). PAH associated with connective tissue disease was the etiology in $\sim 30 \%$ of patients in all three groups. All but 14 patients were in WHO Functional Class II or III; 13 Class IV patients, and 1 Class I patient was enrolled in error. The composite primary end point for the trial was the time from the initiation of treatment to the first event related to $\mathrm{PAH}$ (worsening of $\mathrm{PAH}$, initiation of treatment with intravenous or subcutaneous prostanoids, lung transplantation, or atrial septostomy) or death from any cause to the end of follow-up. Worsening of PAH was defined as (1) a decrease in the 6MWD of at least 15\% from baseline, confirmed by a second 6MWD test performed within 2 weeks of the first; (2) worsening of symptoms of PAH; (3) the need for additional treatment for $\mathrm{PAH}$, and all three of these criteria had to be met. Worsening symptoms of PAH included at least one of the following: a change from baseline to a higher WHO functional class (or no change in patients who were in
WHO Functional Class IV at baseline) and the appearance or worsening of signs of right heart failure that did not respond to oral diuretic therapy. A composite prespecified secondary end point was death or hospitalization due to PAH. The sponsor, Actelion Pharmaceuticals, designed the study and performed the statistical analysis, but all authors had access to the data. The sample size was prospectively re-estimated at 3 months before the end of recruitment and the sample size increased to account for lower-than-expected event rate. The end of the study was declared when the predefined number of endpoints was reached.

Ninety-four patients (12.7\%) who did not have a primary end point discontinued study drug prematurely and were censored at the time of discontinuation from the primary end point analysis. A total of 287 patients over a median follow-up period of 115 weeks had a primary end point, 116 (46.4\%) placebo, 95 patients (38\%) in the $3 \mathrm{mg}$, and 76 patients (31.4\%) in the $10 \mathrm{mg}$ macitentan group. Worsening of pulmonary hypertension was the most frequent primary end point. The hazard ratio for the primary end point for the $3 \mathrm{mg}$ macitentan dose versus placebo was $0.70,97.5 \%$ confidence interval 0.52 to $0.96, p=0.01$ by the log-rank test, and for the $10 \mathrm{mg}$ dose $0.55,97.5 \%$ confidence interval 0.39 to $0.76, p<0.001$. Analyses performed to account for premature discontinuation of treatment were consistent with the primary analysis. Death or hospitalization for $\mathrm{PAH}$ occurred in 84 placebo patients (33.6\%), 65 patients (26.0\%) in the $3 \mathrm{mg}$, and 50 patients (20.7\%) in the $10 \mathrm{mg}$ macitentan group, hazard ratio for $3 \mathrm{mg}$ macitentan dose versus placebo $0.67,97.5 \%$ confidence interval 0.46 to 0.97 , $p=0.01$, hazard ratio for the $10 \mathrm{mg}$ dose versus placebo $0.50,97.5 \%$ confidence interval 0.34 to $0.75, p<0.001$, by the log-rank test. Changes in mean 6MWD at 6 months were small but statistically significant, a decrease of $9.4 \mathrm{~m}$ in the placebo group, an increase of $7.4 \mathrm{~m}$ in the $3 \mathrm{mg}$ macitentan group, and an increase of $12.5 \mathrm{~m}$ in the $10 \mathrm{mg}$ group. In a subset of patients who underwent right heart catheterization at 6 months, there was a significant reduction in pulmonary vascular resistance and increase in cardiac output on both the $3 \mathrm{mg}$ and $10 \mathrm{mg}$ macitentan groups. There was no significant difference in adverse event rates between the three groups, including incidence of edema and aminotransferase elevation. Adjudicated deaths due to $\mathrm{PAH}$ during the double-blind interval or within 4 weeks after randomized treatment had ended occurred 14 patients (5.6\%) in the placebo group, 14 (5.6\%) in the $3 \mathrm{mg}$, and 7 (2.9\%) in the $10 \mathrm{mg}$ macitentan group, hazard ratio 0.44 for placebo versus macitentan $10 \mathrm{mg}$, confidence interval 0.16 to 1.25 , $p=0.07$

In the placebo group, 154 (61.8\%) of total patients were on background PAH therapy and 95 patients (38.2\%) naive to treatment, while in the $10 \mathrm{mg}$ macitentan group 154 patients $(63.6 \%)$ had received other PAH treatment, 88 patients (36.4\%) naïve. The predominant background treatment was a PDE-5 inhibitor, $60 \%$ in all three study groups. Although less patients in the macitentan $10 \mathrm{mg}$ group reached an end point compared with placebo, 6MWD 
improvement, while statistically significant, may not have been biologically meaningful. While there was a trend, whether due to sample size, duration of follow-up, or other variable, survival benefit was not demonstrated. Subjects randomly assigned to the placebo group who experienced a nonfatal event were allowed to have open-label macitentan, complicating assessment of end point findings. ${ }^{11}$ Summarizing, there is a signal in SERAPHIN that combination therapy in PAH may be beneficial, in the near two-thirds of patients in the $10 \mathrm{mg}$ macitentan group who were already receiving background PAH therapy, and in most a PDE-5 inhibitor.

\section{AMBITION}

While SERAPHIN and other trials assessed effect of an agent added to background therapy, the AMBITION trial ${ }^{4}$ assessed the impact of de novo combination therapy on long-term outcome. The AMBITION trial was a multicenter doubleblind trial in which WHO Group 2 and 3 patients were randomized in a 2:1:1 ratio within PAH strata (idiopathic or heritable versus nonidiopathic) to receive ambrisentan and tadalafil or ambrisentan plus placebo or tadalafil plus placebo. The nonidiopathic strata included PAH associated with connective tissue disease, repaired congenital systemicto-pulmonary shunts, human immunodeficiency virus infection, or drug use or toxin exposure-the same as SERAPHIN, except that enrolled subjects had either not received previous treatment with an approved therapy for PAH or had received treatment for less than 14 days and had not received any approved therapy for PAH within 7 days before enrollment. Ambrisentan and tadalafil were increased to target doses of $10 \mathrm{mg}$ and $40 \mathrm{mg}$, respectively. The composite primary end point in a time-to-event analysis was all-cause mortality, hospitalization for worsening pulmonary hypertension (any hospitalization for PAH, lung or heart and lung transplantation, atrial septostomy, or initiation of prostanoid therapy), disease progression (15\% or more decrease in 6MWD combined with WHO Functional Class III or IV symptoms at two consecutive visits separated by at least 14 days), or unsatisfactory long-term clinical response (any decrease in 6MWD at two consecutive visits separated by at least 14 days and WHO Class III symptoms at two clinic visits separated by at least 6 months). A total of 610 patients were randomized, 5 of whom did not receive study medication. Of the 605 participants receiving medication, 310 were randomized to receive combination therapy, 152 ambrisentan monotherapy, and 151 tadalafil monotherapy. A blinded review performed by the steering committee 6 months into randomization revealed higher-than-expected prevalence of risk factors for left ventricular diastolic dysfunction, and a decision was made to amend eligibility criteria to exclude individuals with three or more risk factors for LV diastolic dysfunction. An adjudicated primary event was required in 105 randomized participants in the primary analysis set to provide $97 \%$ power to identify a $53 \%$ decrease in the risk of clinical failure in the combination group compared with the pooled monotherapy groups combined. The primary analysis set comprised 500 patients fulfilling the amended entry criteria, 253 combination-therapy patients, 126 ambrisentan monotherapy, and 121 tadalafil monotherapy participants. Most patients had either idiopathic $\mathrm{PAH}$ or $\mathrm{PAH}$ associated with connective tissue disease; $69 \%$ of patients were WHO Functional Class III. The mean PA pressure for the study population was $48.7 \mathrm{~mm} \mathrm{Hg}$, and mean 6MWD was $352.6 \mathrm{~m}$. The mean duration in this event-driven trial was 609 days.

A total of 64 participants (13\%) withdrew from the study before having a primary end point event, of whom 8 subsequently had an event that was included in the primary end point analysis. A primary event rate occurred before the final assessment visit in a total of 123 patients, $46(18 \%)$ in the combination group and 77 (31\%) in the pooled monotherapy group, of which 43 (34\%) were ambrisentan and 34 (28\%) in the tadalafil monotherapy group, hazard ratio 0.50 , confidence interval 0.35 to $0.72, p<0.001$ for the combination group compared with the pooled monotherapy group, and comparisons between the combination group and the individual monotherapy groups also demonstrated a statistically significant advantage to combination therapy. Hospitalization for worsening PAH showed the largest difference between combination therapy compared with the pooled cohort, 4 versus $12 \%$. There was no significant difference in mortality, $9(4 \%)$ in the combination group versus $8(3 \%)$ in the pooled monotherapy cohort. Among prespecified secondary end points, NTproBNP was $17 \%$ lower among combination therapy patients compared with pooled monotherapy, $p<0.001$, and 6MWD increased by a median of $48.98 \mathrm{~m}$ in the combination group, compared with $23.8 \mathrm{~m}$ in the pooled monotherapy group, $p<0.001$. Peripheral edema occurred in $48 \%$ of the combination group. Adverse reactions leading to discontinuation of study medication occurred in $12 \%$ of the combination group, $11 \%$ of the ambrisentan monotherapy group, and $12 \%$ of the tadalafil monotherapy group.

The AMBITION trial is the first randomized trial to demonstrate a benefit targeting multiple vasoconstrictive pathways in patients with $\mathrm{PAH}$ who are naive to therapy. Combination treatment benefit was primarily a result of lower rate of hospitalization for worsening PAH, compared with monotherapy. Separation of the log-rank curves for the primary end point was first noted at week 24 . The significance of a tendency to convergence of the curves at 144 weeks could not be analyzed due to the small number of remaining patients, but may indicate waning of the beneficial effect of this combination therapy over longer intervals of observation. Among other limitations, while 6MWD was better and NTproBNP lower in the combination group compared with monotherapy, there was no improvement in WHO functional class at week 24, and the trial design did not allow for the assessment of whether response rates in either of the monotherapy groups would have improved had subjects receiving one agent been switched to the other agent. Originally Food and Drug Administration (FDA) approved in 2007 to improve exercise tolerance, the results of the AMBITION trial resulted in the additional indication for ambrisentan of reducing disease progression and hospitalization when given in combination with tadalafil. The AMBITION trial nevertheless provided compelling evidence 
that among patients with $\mathrm{PAH}$, combination therapy with ambrisentan and tadalafil is superior to either agent alone, but does not necessarily allow for extrapolation to other medications in the same class.

\section{GRIPHON}

The GRIPHON trial ${ }^{5}$ was an event rate-driven, randomized, double-blind, placebo-controlled trial of the IP receptor agonist selexipag in 1,156 patients with $\mathrm{PAH}$, the characteristics of which were the same as for SERAPHIN and AMBITION. The trial results would lead to FDA approval of selexipag in 2015 to delay disease progression and reduce the risk of hospitalization for PAH. At study, entry patients were required to have a pulmonary vascular resistance of at least 5 Wood units, a 6MWD of 50 to $450 \mathrm{~m}$, and could be enrolled if receiving no therapy, an endothelin receptor antagonist, a PDE-5 inhibitor, or both, on a stable dose of PAH medications for the 3 months preceding randomization. Patients were randomized 1:1 to receive either selexipag or placebo. During a 12-week dose adjustment phase, selexipag was started at the usual initial dose of $200 \mu \mathrm{g}$ twice daily and increased weekly in increments of $200 \mu \mathrm{g}$ twice daily to a maximum dose of $1,600 \mu \mathrm{g}$ twice daily as tolerated until unmanageable side effects associated with prostanoid use, such as jaw pain, headache, or nausea. The end of the treatment period of the study occurred at the end of the study for patients who did not have a primary end point, at the occurrence of a primary end point, or prematurely for various reasons, such as an adverse event. When the prespecified number of primary end points had been reached, the end of the study was declared. The primary end point was the composite of death or a complication related to PAH, including progression of disease that required hospitalization, initiation of parenteral prostanoid or long-term oxygen therapy, or the need for atrial septostomy or lung transplantation, or placement on the lung transplantation list. Primary events were adjudicated by an independent critical-event committee. Twenty months after the study was initiated, a blinded review of 154 patients indicated that more patients than expected were receiving background therapy, and the prespecified target hazard ratio was changed from 0.57 to 0.65 to reflect the lower anticipated treatment effect. The anticipated event rate was changed from 202 to 331, requiring an increase in enrollment to 1,150. The primary end point analysis was for on-treatment participants, with follow-up data censored at the time selexipag or placebo was discontinued. Changes from baseline for 6MWD test and NTproBNP were analyzed at week 26.

Enrollment encompassed 1,156 patients at 181 centers in 39 countries from 2009 to 2013, 574 assigned to selexipag, and 582 to placebo. Mean follow-up duration for the selexipag group was 70.7 weeks and 63.7 weeks for the placebo group. Of 351 (30\%) participants who discontinued selexipag or placebo after a nonfatal primary end point event, 170 provided consent for follow-up during the post-treatment interval (59 selexipag and 111 placebo). Of 218 patients who discontinued study drug before experiencing a primary end point event, 80 provided consent for follow-up during the post-treatment interval ( 54 selexipag and 26 placebo). Vital status was known for 1,101 patients (95.2\%) at the end of the study.

A primary event occurred in 397 patients overall, 155 patients $(27.0 \%)$ in the selexipag, and 242 patients (41.6\%) in the placebo group, hazard ratio $0.60,99 \%$ confidence interval 0.46 to $0.78, p<0.001$. Progression of disease and hospitalization accounted for $81.9 \%$ of primary events. Sensitivity analyses conducted to account for early withdrawals and for the recalculation of the anticipated hazard ratio were in accord with the primary analysis. The effect of selexipag was consistent across a low-dose, medium-dose, and high-dose stratum, and $42.9 \%$ of selexipag patients were in the highdose stratum of 1,200 to $1,600 \mu$ twice daily. At week 26, 6minute distance had increased by $4.0 \mathrm{~m}$ from baseline in the selexipag group and decreased by $9 \mathrm{~m}$ in the placebos group, $p=0.003$, confidence interval 1 to 24 . There was essentially no significant change in functional class between the selexipag and placebo groups. Exploratory analyses included a hazard ratio of $0.70, p=0.003$, in favor of selexipag over placebo for death due to $\mathrm{PAH}$ or hospitalization due to worsening PAH; $87.4 \%$ of these events were hospitalization for worsening $\mathrm{PAH}$. At the end of the study, death from any cause had occurred in 100 patients $(17.4 \%)$ in the selexipag and 105 patients in the placebo group (18.0\%), hazard ratio $0.97, p=0.42$. At 26 weeks, NTproBNP level had decreased by $34.5 \mathrm{ng} / \mathrm{L}$ in the selexipag group, compared with an increase of $18.0 \mathrm{ng} / \mathrm{L}$ in the placebo group, $p<0.001$.

In a prespecified subgroup analysis of patients on both an endothelin receptor antagonist and a PDE-5 inhibitor, the impact of selexipag versus placebo on the primary end point was nearly the same as had been seen for the study as a whole, hazard ratio 0.69 , statistic not published, but in a forest plot provided in the published supplement, the $99 \%$ confidence interval does not cross the line of unity. The effect of selexipag added to a PDE-5 inhibitor alone also appears to have been significant by the $99 \%$ confidence interval; however, for selexipag added to an endothelin receptor antagonist, although the hazard ratio reflected a reduction in primary event rate, 0.62 , the confidence interval crossed the line of unity, indicating not statistically significant.

Similar to findings with the addition of an endothelin receptor antagonist to a PDE-5 inhibitor, adding selexipag to background therapy resulted in improvement in a similar combined end point, and GRIPHON is the first randomized trial demonstrating that triple-drug therapy in $\mathrm{PAH}$ is effective.

\section{Other Trials}

The PATENT-1 trial $^{12}$ assessed impact of the guanylate cyclase agonist riociguat on 6MWD as the primary end point in $\mathrm{PAH}$ patients but in addition to those randomization WHO Group 1 PAH patients in the foregoing trials, included patients with portopulmonary hypertension. Patients on PDE-5 inhibitors and parenteral prostanoids were excluded. Approximately $45 \%$ of patients were on background treatment with an endothelin receptor antagonist, and 6\% on an oral prostanoid. In 113 patients on an endothelin receptor 
antagonist, riociguat resulted in a mean increase in 6MWD of $23 \mathrm{~m}, 95 \%$ confidence interval 1 to 48 . In 18 patients on an oral prostanoid, riociguat was associated with a mean 6MWD increase of $56 \mathrm{~m}, 95 \%$ confidence interval 38 to 173 .

Oral treprostinil was approved in 2013 to improve exercise capacity primarily on the basis of three FREEDOM trials. ${ }^{13-15}$ The FREEDOM-C trial ${ }^{13}$ assessed the effect of oral treprostinil versus placebo on 6MWD after 16 weeks of treatment in 106 patients receiving concomitant endothelin receptor antagonist alone, 88 patients receiving concomitant PDE-5 inhibitor alone, and 156 patients receiving both endothelin receptor antagonist and PDE-5 inhibitor therapy, 350 patients total, 174 patients randomized to oral treprostinil compared with 176 placebo patients. The majority of enrolled subjects were in functional Class II or III. Most of the patients randomized to oral treprostinil started at either $0.5 \mathrm{mg}$ or $1 \mathrm{mg}$ twice daily, as smaller dose preparations did not become available until late in the trial. Thirty-nine patients (22\%) receiving oral treprostinil and 24 patients (14\%) receiving placebo discontinued the study drug. In the 39 oral treprostinil patients discontinuing drug, 25 were for any of 13 adverse events ranging from headache to emesis, 7 for clinical worsening, 2 lost to follow-up, 1 lung transplantation, 1 withdrawal of consent, 1 protocol violation, and 2 "other." Reasons for drug discontinuation in the placebo group were 8 adverse events, 8 for clinical worsening, 1 death, 1 lung transplant, 1 withdrawal of consent, 1 protocol violation, and 4 "other." There were a total of six deaths, three in each arm. The median change in 6MWD at week 16 for the treprostinil was $14.5 \mathrm{~m}$ and for the placebo group $4.8 \mathrm{~m}$, median difference $11 \mathrm{~m}, p=0.07$, not significant, but was significant when combined with Borg dyspnea score and dyspnea fatigue index score. Possible reasons for the lack of a significant increase in 6MWD included the high oral treprostinil discontinuation rate and the relatively short duration of the trial in patents already on background therapy. The FREEDOM-M ${ }^{14}$ trial evaluated oral treprostinil monotherapy in PAH patients starting at a lower dose of drug, $0.25 \mathrm{mg}$, than was employed in the majority of FREDDOM-C patients and demonstrated significantly improved 6MWD in the oral treprostinil arm, and therefore the FREEDOM-C2 trial $^{15}$ was performed for patients on background PAH therapy, with the lower starting dose employed successfully in FREEDOMM. The mean oral treprostinil dose at 16 weeks was $3.1 \pm 1.9 \mathrm{mg}$. As had been seen in FREEDOM-C, the median difference in 6MWD was $10.0 \mathrm{~m}$, not reaching statistical significance, $p=0.089$. Notably, the changes in 6MWD in FREEDOM-C and C2 and GRIPHON are not dissimilar.

Among other factors, issues of tolerability at initial starting dose, trial duration, and the importance of an assessment of end points led to the design of FREEDOM-EV ${ }^{16}$ a doubleblind, placebo-controlled, event-driven trial of oral treprostinil added to background monotherapy in PAH, was initiated in 2012, enrollment completed during the summer of 2018 , and the key data of which were presented at the Pulmonary Vascular Research Institute Annual World Congress in January 2019. Background therapy included $72 \%$ of patients on PDE-5 inhibitor and $28 \%$ on an endothelin receptor antagonist. A total of $63 \%$ of enrolled patients were Functional Class
II, mean time from PAH diagnosis 0.54 years; to the author's knowledge, the median PA pressure has not been reported. By 24 weeks after randomization, the median oral treprostinil dose was $3.5625 \mathrm{mg}$ three times daily. Over the course of the trial $19 \%$ of patients randomized to oral treprostinil and $4 \%$ of placebo participants discontinued randomized treatment for adverse effects. Oral treprostinil decreased the risk of adjudicated clinical worsening events by $26 \%$ compared with placebo, $p=0.0391$, largely due to a $61 \%$ decrease in the risk of in disease progression, $p=0.0002$. In addition, in the $89 \%$ of participants for whom data were available at the time of presentation, oral treprostinil was associated with a $37 \%$ decrease in mortality compared with placebo, $p=0.0324$ (includes data accrued during an openlabel extension study). If proven at the conclusion of the trial, and if it withstands peer review, this finding will be the second treatment to demonstrate survival benefit in PAH.

A group of investigators many of whom participated in the FREEDOM trials have proposed a strategy for the use of oral treprostinil ${ }^{17}$ several components of which may be as practically applicable to any oral IP receptor as to a prostanoid. Abbreviated guidelines are as follows: (1) administer oral treprostinil three times daily and take with food; (2) start $0.125 \mathrm{mg}$ or $0.25 \mathrm{mg}$ in larger patients; (3) uptitrate slowly, less than weekly if necessary and in small dose increments, and decrease for adverse effects (and by slowly, the author would add as slowly as necessary to make adverse effects tolerable), consider step dosing, that is, increasing by one of the three doses daily; (4) treat with nonnarcotic antidiarrheal and antiemetic drugs if necessary; (5) treat headache with acetaminophen, rarely advance to tramadol, consider a pain-modifying drug such as amitriptyline or gabapentin; (6) apply ample amounts of reassurance. With or without an IP receptor agonist, an overriding guideline is that combination therapy is best applied by practitioners experienced in the management of $\mathrm{PAH}$, with a staff of compassionate clinic associates without whom these patients would be adrift.

\section{Summary and Thoughts}

In the author's own experience, the first "substantive" use of an oral IP receptor occurred 5 years ago in a patient with PAH associated with Takayasu's arteritis, on a PDE-5 inhibitor and epoprostenol, who had sustained a third methicillin-resistant Staphylococcus aureus (MRSA) infection from parenteral prostanoid access. Three years earlier when admitted for worsening clinical status, the patient experienced an adverse reaction to an endothelin receptor antagonist, and was started on epoprostenol. At the time of the third MRSA infection, the epoprostenol dose was $50 \mu \mathrm{g} / \mathrm{kg} / \mathrm{min}$. We made a decision to wean from epoprostenol and simultaneously start and uptitrate oral treprostinil. As the patient had become accustomed to epoprostenol side effects, we were able to rapidly increase oral treprostinil to $10 \mathrm{mg}$ TID-an exception to the start low and go slow rule. Currently, the patient is on $27.5 \mathrm{mg}$ TID oral treprostinil with minimal prostanoid side effects, averages $350 \mathrm{~m}$ on 6MWD testing, and has not been rehospitalized for 
PAH since 2014. Significant experience has been gained in transitioning PAH patients from parenteral to oral agents, from one or more oral agents to others. ${ }^{18}$ In particular, transitioning patients from parenteral prostanoid to oral treprostinil or selexipag must be an individualized process based on multiple patient factors.

All PAH practitioners have experience with oral IP receptor agonist discontinuation for adverse effects, in spite of close coaching. As was the case with combining a nitric oxide pathway drug with an endothelin receptor antagonist, and in view of available findings, adding a third medication is reasonable when one is treating disease associated with far-less-than-normal survival. The improvement in survival odds to a median of 6 years in the past decade, compared with 2.5 to 3 years in the $1980 \mathrm{~s},{ }^{1}$ is likely to be due in part to the availability and combined use of PAH drug therapies. The practical application of combination therapy in PAH has recently been summarized. ${ }^{19}$ Among other useful points is that all PAH patients may not tolerate the simultaneous initiation of a PDE-5 inhibitor and an endothelin receptor antagonist, that is, combination therapy may need to be initiated in a stepped approach. For those patients intolerant of oral IP receptor agonists, for example, in whom an oral IP receptor was initiated because of a decrease in 6MWD or an increase in NTproBNP as opposed to progression to functional Class IV, the clinician can consider a trial of either inhaled treprostinil or iloprost to improve functional capacity. End point-driven data for the addition of inhaled prostanoids are limited, as is the case for the addition of an oral agent to parenteral prostanoid. ${ }^{20}$ However, economic analyses confirm that over the past decade a substantial number of PAH patients have received oral therapies added to a prostacyclin, from $61.2 \%$ in 2010 to $79.2 \%$ in $2015 .^{21}$ The PAH practitioner may also see patients with what appears to be marked systemic vasodilation from PAH drugs, in which patients experience not only the usual adverse side effects but also worsening functional capacity, when the patient has reached the limit of pulmonary vasodilation that can occur in plexogenic pulmonary arteries and systemic effects of PAH medications exceed pulmonary, In these situations, it may be possible to decrease a drug dose or discontinue a drug. For example, in a new idiopathic $\mathrm{PAH}$ patient with functional Class IV presentation, the patient acutely decompensated after right heart catheterization with nitric oxide testing, stabilized in the recovery room with reinstitution of nitric oxide, was then started on parenteral prostanoid, and subsequently during the same hospitalization on combination PDE-5 inhibitor/endothelin receptor antagonist therapy. Following institution of combination oral therapy, parenteral prostanoid was weaned and discontinued due to intolerable side effects. Recently, the patient has been started on an oral IP receptor agonist using a low-dose-first approach. What $\mathrm{PAH}$ patients hope for, and $\mathrm{PAH}$ practitioners, is a cure, but a more effective means of bringing about regression intimalmedial proliferation would be wonderful.

In conclusion, dual-drug treatment in PAH can be considered largely proven in WHO Group $1 \mathrm{PAH}$, with the exception of patients with portopulmonary hypertension.
It can be argued persuasively that two-drug PAH treatment has become the rule in PAH clinics, and many patients on triple-drug therapy. Findings from studies have "caught up" with this approach. With promising strategies under investigation to interrupt the proliferative, neoplastic-like nature of the disease, ${ }^{1,22}$ patients and PAH care providers can hope to see continued improvement in outcome, perhaps hopedfor eradication of this disease.

Conflicts of Interest

No conflicts to disclose.

\section{Acknowledgments}

The author acknowledges the tireless contributions of Kim Jackson, RN, Kendra Griffin, and Mary Jane Cowherd, $\mathrm{PA}-\mathrm{C}$, to the care and well-being of all the pulmonary hypertension patients we see at UK Medical Center.

\section{References}

1 Thenappan T, Ormiston ML, Ryan JJ, Archer SL. Pulmonary arterial hypertension: pathogenesis and clinical management. BMJ 2018; 360:j5492

2 Badesch DB, Raskob GE, Elliott CG, et al. Pulmonary arterial hypertension: baseline characteristics from the REVEAL Registry. Chest 2010;137(02):376-387

3 Pulido T, Adzerikho I, Channick RN, et al; SERAPHIN Investigators. Macitentan and morbidity and mortality in pulmonary arterial hypertension. N Engl J Med 2013;369(09):809-818

4 Galiè N, Barberà JA, Frost AE, et al; AMBITION Investigators. Initial use of ambrisentan plus tadalafil in pulmonary arterial hypertension. N Engl J Med 2015;373(09):834-844

5 Sitbon O, Channick R, Chin KM, et al; GRIPHON Investigators. Selexipag for the treatment of pulmonary arterial hypertension. $\mathrm{N}$ Engl J Med 2015;373(26):2522-2533

6 Badesch DB, Feldman J, Keogh A, et al; ARIES-3 Study Group. ARIES-3: ambrisentan therapy in a diverse population of patients with pulmonary hypertension. Cardiovasc Ther 2012;30(02): 93-99

7 McLaughlin VV, Badesch DB, Delcroix M, et al. End points and clinical trial design in pulmonary arterial hypertension. J Am Coll Cardiol 2009;54(1, Suppl):S97-S107

8 McLaughlin V, Channick RN, Ghofrani HA, et al. Bosentan added to sildenafil therapy in patients with pulmonary arterial hypertension. Eur Respir J 2015;46(02):405-413

9 Barst RJ, Rubin LJ, Long WA, et al; Primary Pulmonary Hypertension Study Group. A comparison of continuous intravenous epoprostenol (prostacyclin) with conventional therapy for primary pulmonary hypertension. N Engl J Med 1996;334(05):296-301

10 Hemnes AR. Using omics to understand and treat pulmonary vascular disease. Front Med (Lausanne) 2018;5:157

11 To the editor. Macitentan and pulmonary arterial hypertension. 1N Engl. J Med 2014;370:81-83

12 Ghofrani H-A, Galiè N, Grimminger F, et al; PATENT-1 Study Group. Riociguat for the treatment of pulmonary arterial hypertension. N Engl J Med 2013;369(04):330-340

13 Tapson VF, Torres F, Kermeen F, et al. Oral treprostinil therapy for the treatment pulmonary arterial hypertension in patients receiving background endothelin antagonist and phosphodiesterase type 5 inhibitor therapy (the FREEDOM-C study): a randomized controlled trial. Chest 2012;142(06):1383-1390

14 Jing ZC, Parikh K, Pulido T, et al. Efficacy and safety of oral treprostinil monotherapy for the treatment of pulmonary arterial hypertension: a randomized, controlled trial. Circulation 2013; 127(05):624-633 
15 Tapson VF, Jing ZC, Xu KF, et al; FREEDOM-C2 Study Team. Oral treprostinil for the treatment of pulmonary arterial hypertension in patients receiving background endothelin receptor antagonist and phosphodiesterase type 5 inhibitor therapy (the FREEDOMC2 study): a randomized controlled trial. Chest 2013;144(03): 952-958

16 Clinical Trials.gov. Trial of the early combination of oral treprostinil with background oral monotherapy in subjects with pulmonary arterial hypertension.

17 Rahaghi FF, Feldman JP, Allen RP, et al. Recommendations for the use of oral treprostinil in clinical practice: a Delphi consensus project pulmonary circulation. Pulm Circ 2017;7(01): 167-174

18 Sofer A, Ryan MJ, Tedford RJ, Wirth JA, Fares WH. A systematic review of transition studies of pulmonary arterial hypertension specific medications. Pulm Circ 2017;7(02):326-338
19 Burks M, Stickel S, Galiè N. Pulmonary arterial hypertension: combination therapy in practice. Am J Cardiovasc Drugs 2018;18 (04):249-257

20 Simonneau G, Rubin LJ, Galiè N, et al; PACES Study Group. Longterm sildenafil added to intravenous epoprostenol in patients with pulmonary arterial hypertension. J Heart Lung Transplant 2014;33(07):689-697

21 Burger CD, Pruett JA, Lickert CA, Berger A, Murphy B, Drake W III. Prostacyclin use among patients with pulmonary arterial hypertension in the United States: a retrospective analysis of a larger health care claims database. J Manag Care Spec Pharm 2018;24(03): 291-302

22 Dai Z, Zhu MM, Peng Y, et al. Endothelial and smooth muscle cell interaction via FoxM1 signaling mediates vascular remodeling and pulmonary hypertension. Am J Respir Crit Care Med 2018; 198(06):788-802 